\title{
A case report of successful survival after tracheal intubation and IMV in elderly patient of COVID-19 with hypertension
}

\author{
ruijing XIAO ${ }^{1}$, Chaogui Zhang ${ }^{2}$, Fengling Luo ${ }^{3}$, Yingcheng Zheng ${ }^{3}$, Li Liu ${ }^{4}$, Hua Yang ${ }^{2}$, \\ and Wanhong $\mathrm{Liu}^{3}$ \\ ${ }^{1}$ Affiliation not available \\ ${ }^{2}$ University Hospital of Hubei University for Nationalities \\ ${ }^{3}$ Wuhan University \\ ${ }^{4}$ The University of Hong Kong,
}

April 28, 2020

\begin{abstract}
Coronavirus disease 2019 (COVID-19) caused by severe acute respiratory syndrome coronavirus (SARS-CoV)-2 infection has become a pandemic cause of viral pneumonia. Elderly patients with chronic comorbidities are susceptible to SARS-CoV-2, and are more likely to have complications that can lead to severe or even fatal consequences. It is important to seek the appropriate effective treatment for these susceptible persons. Here we report a case of elderly COVID-19 patient with hypertension, which was a member of the familial cluster COVID-19 in Enshi City, Hubei Province, China. He successfully survived after removal of tracheal intubation and inhalation of the invasive mechanical ventilation (IMV). This report aims to provide an inspiration for the prevention and treatment of critical COVID-19 cases.
\end{abstract}

\section{Introduction}

Coronavirus disease 2019 (COVID-19) caused by severe acute respiratory syndrome coronavirus (SARS$\mathrm{CoV}$ )-2 infection has become a pandemic cause of viral pneumonia (Lescure FX et al, 2020; Wang C et al, 2020), leading to over 2 million cases with more than 146 thousands death up to April $18^{\text {th }}, 2020$. Studies have shown that pediatric patients present mostly mild and moderate cases (Qiu H et al, 2020). In contrast, Elderly patients with comorbidities are susceptible to SARS-CoV-2, and are more likely to have complications that can lead to severe or even fatal consequences (Chen N et al, 2020). SARS-CoV-2 is highly contagious, especially in the case of family cluster transmission (Chan JF et al, 2020). Early and effective isolation of diagnosed COVID-19 patients will reduce the chance of infection for other family members.

Here, we report a case of family cluster COVID-19 with high-risk characteristics: older age, history of hypertension, which successfully survived after removal of tracheal intubation and invasive mechanical ventilation (IMV) in Enshi City, Hubei Province, China. Due to the current poor prognosis of tracheal intubation and IMV in patients with COVID-19, the successful survival of this case has given great encouragement and confidence to clinicians and similar patients. We actively discussed the relevant content of this case with a view to inspiring the prevention and treatment of critical COVID-19 cases.

\section{Case presentation}

The patient was a 60-year-old COVID-19 male with hypertension who had undergone long-term contact with a confirmed COVID-19 patient (his father, who had passed away on Feb 10, 2020). He was admitted to the hospital in Enshi City as a moderate COVID-19 case on Feb 12, 2020 after initial diagnoses: productive coughing, positive SARS-CoV-2 nucleic acid test, and CT results (infectious lesions of the right and left lower 
lobe, ground glass changes) (Figure 1A). Blood test displayed an increased neutrophil percentage (73.5\%) and a decreased lymphocyte percentage $(15.6 \%)$ (Table1). In view of continuous fever, Computed Tomography (CT) was reviewed on the 8th day (Feb 16, 2020). The image presented multifocal ground glass opacities with consolidation of significantly larger range and higher density compared to previous observations. In addition, there were several fibrous calcifications in the lungs (Figure 1B).

On the 12th day of admission, the patient developed dyspnea and a non-invasive mechanical ventilation (NIV) was used to assist breathing. On the 13th day, the patient's condition further deteriorated $\left(\mathrm{SaO}_{2} 49 \cdot 2 \%\right)$ (Table1). Tracheal intubation and invasive mechanical ventilation (IMV) were performed immediately, $\mathrm{SaO}_{2}$ subsequently rose to $100 \%$. After intubation, the patient developed a second-degree atrioventricular block of the heart and an increase in blood pressure $(190 / 100 \mathrm{mmHg})$, which returned to normal after symptomatic treatment of atropine and sodium nitroprusside. Meanwhile, he continued to be treated for anti-infection, antiviral, nutritional support, stomach protection and correction of electrolyte disorders. On the 5th day after intubation (Feb 28, 2020), the patient's liver function became worse (ALT 239.0 U/L, AST $168 \mathrm{U} / \mathrm{L}$ and $\gamma$-GT $108 \mathrm{U} / \mathrm{L}$ ) (Table 1). With the enhancement of liver protection treatment, patients' liver function did not deteriorate further.

On the 20th day, the patient was conscious. Tracheal intubation was removed and NIV was used to assist breathing ( $\mathrm{SaO} 2100 \%)$. After the removal of intubation, CT re-examination showed ground glass opacities with consolidation in the lungs, the range was larger and the density of some lesions was higher than before (Figure 1C). On the 35th day the patient satisfied discharge criteria and was maintained in isolation. The last lung CT image showed that the lesion was absorbed compared to previous observations (Figure 1D). During the isolation period, treatments such as oxygen inhalation, liver protection, phlegm elimination, and anti-infection were continued. On Mar 22, 2020, patient SARS-CoV-2 IgG antibody tested positive.

\section{Discussion}

We report a critical COVID-19 case in Enshi City who successfully survived after tracheal intubation and IMV treatment. A 60-year-old patient, a history of hypertension, persistent recurrent fever after admission, the results of blood tests and CT results all indicated that he could progress to critical status (Shi $\mathrm{H}$ et al, 2020; Zhang G et al, 2020). Due to deterioration, NIV treatment was performed on Feb 23, 2020, but the breathing condition was not improved. The next day (Feb 24, 2020), tracheal intubation and IMV were performed. Subsequently, the breathing condition was greatly improved, after which the $\mathrm{SaO}_{2}$ remained at $99-100 \%$. We believed that early and timely assisted breathing interventions have played a better role in curbing the disease progression of this patient.

Hypertension and diabetes are reported to be the most common comorbidities of COVID-19, followed by cardiovascular disease (Huang $\mathrm{C}$ et al, 2020; Wang D et al, 2020). Wang FS et al. reported (Zhang Cet al, 2020) that $2-11 \%$ of patients with COVID-19 have liver comorbidities, while $14-53 \%$ of patients with abnormal levels of ALT and AST, and the critical COVID-19 patients seem to have a higher rate of liver dysfunction. In this case, the patient had abnormal heart conditions, increased blood pressure, and hepatic dysfunction after intubation. However, they can be gradually improved via active and effective symptomatic treatment.

In general, for elderly patients with comorbidities, in the case of critical symptoms such as dyspnea and extreme decline of $\mathrm{SaO}_{2}$, the active control of the progress of comorbidities, and early and timely assisted respiratory interventions may prevent the disease from progressing to adverse outcomes. In addition, for patients of COVID-19 with impaired liver function, stricter surveillance and targeted treatment are needed. The successful recovery of this patient has given great encouragement and confidence to clinicians. However, multiple factors contribute to COVID-19 pathology and its composite endpoint. More related research and reports are needed in the future.

\section{Ethical Statement}

All studies were approved by and performed according to the guidelines of the ethics committee at University 
Hospital of Hubei University for Nationalities and Wuhan University (China).

\section{Data Availability Statement}

All data, models, and code generated or used during the study appear in the submitted article.

\section{Acknowledgment}

We thank these parents involved in the study. This work was supported by grants from the Central Universities of China and the Translational Medical Research Fund of Wuhan University School of Medicine.

\section{Conflict of interest}

No conflicts of interest were declared.

\section{References}

Chen N, Zhou M, Dong X, Qu J, Gong F, et al. (2020). Epidemiological and Clinical Characteristics of 99 Cases of 2019 Novel Coronavirus Pneumonia in Wuhan, China: A Descriptive Study. Lancet , 395 (10223):507-513. doi : 10.1016/S0140-6736(20)30211-7.

Chan JF, Yuan S, Kok KH, To KK, Chu H, et al. (2020). A Familial Cluster of Pneumonia Associated With the 2019 Novel Coronavirus Indicating Person-To-Person Transmission: A Study of a Family Cluster.Lancet , 395 (10223):514-523. doi : 10.1016/S0140-6736(20)30154-9.

Huang C, Wang Y, Li X, Ren L, Zhao J, et al. (2020). Clinical features of patients infected with 2019 novel coronavirus in Wuhan, China. Lancet, 395(10223):497-506. doi : 10.1016/S0140-6736(20)30183-5.

Lescure FX, Bouadma L, Nguyen D, Parisey M, Wicky PH,et al. (2020).Clinical and virological data of the first cases of COVID-19 in Europe: a case series. Lancet Infect Dis, pii: S1473-3099(20)30200-0. doi : 10.1016/S1473-3099(20)30200-0.

Qiu H, Wu J, Hong L, Luo Y, Song Q, Chen D. (2020). Clinical and epidemiological features of 36 children with coronavirus disease 2019 (COVID-19) in Zhejiang, China: an observational cohort study.Lancet Infect Dis , pii: S1473-3099(20)30198-5. doi : 10.1016/S1473-3099(20)30198-5.

Shi H, Han X, Jiang N, Cao Y, Alwalid O, Gu J, Fan Y, Zheng C. (2020). Radiological Findings From 81 Patients With COVID-19 Pneumonia in Wuhan, China: A Descriptive Study. Lancet Infect Dis, 20 (4):425-434.doi : 10.1016/S1473-3099(20)30086-4.

Wang C, Horby PW, Hayden FG, Gao GF.(2020). A Novel Coronavirus Outbreak of Global Health Concern. Lancet , 395 (10223):470-473.doi : 10.1016/S0140-6736(20)30185-9.

Wang D, Hu B, Hu C, Zhu F, Liu X, et al. (2020). Clinical Characteristics of 138 Hospitalized Patients With 2019 Novel Coronavirus-Infected Pneumonia in Wuhan, China. JAMA, 323(11):1061-1069. doi : 10.1001/jama.2020.1585.

Zhang C, Shi L, Wang FS. (2020). Liver injury in COVID-19: management and challenges. Lancet Gastroenterol Hepatol, 5(5):428-430. doi: 10.1016/S2468-1253(20)30057-1.

Zhang G, Zhang J, Wang B, Zhu X, Wang Q, Qiu S. (2020). Analysis of clinical characteristics and laboratory findings of 95 cases of 2019 novel coronavirus pneumonia in Wuhan, China: a retrospective analysis. Respiratory Research, 21 (1):74. doi : 10.1186/s12931-020-01338-8.

Tables

Table 1. Analysis of Clinical Characteristics and Laboratory Findings of this patient.

Figure Legends

Figure1. Chest computed tomographic images of this patient of COVID-19 from outpatient department to discharge criteria were met. A. CT image before admission: right lung and left lung 
lower lobe infectious lesions, multifocal ground glass changes. B. CT image on the 8th day after admission (2020.02.19 before tracheal intubation): multiple infectious lesions in the lungs, showing multifocal ground glass opacities with consolidation, the range is significantly larger than before, the density is higher than before, a few fibrous calcifications in the lungs. C. CT image on the 26th day after admission (2020.03.08 after removal of tracheal intubation): Multiple infectious lesions in the lungs show ground glass changes, the range is larger than the previous results and the density of some lesions is higher than before. D. CT image on the 36th day after admission (the second day of isolated observation on 2020.03.18): the absorption of bilateral ground glass opacity compared with the previous image.
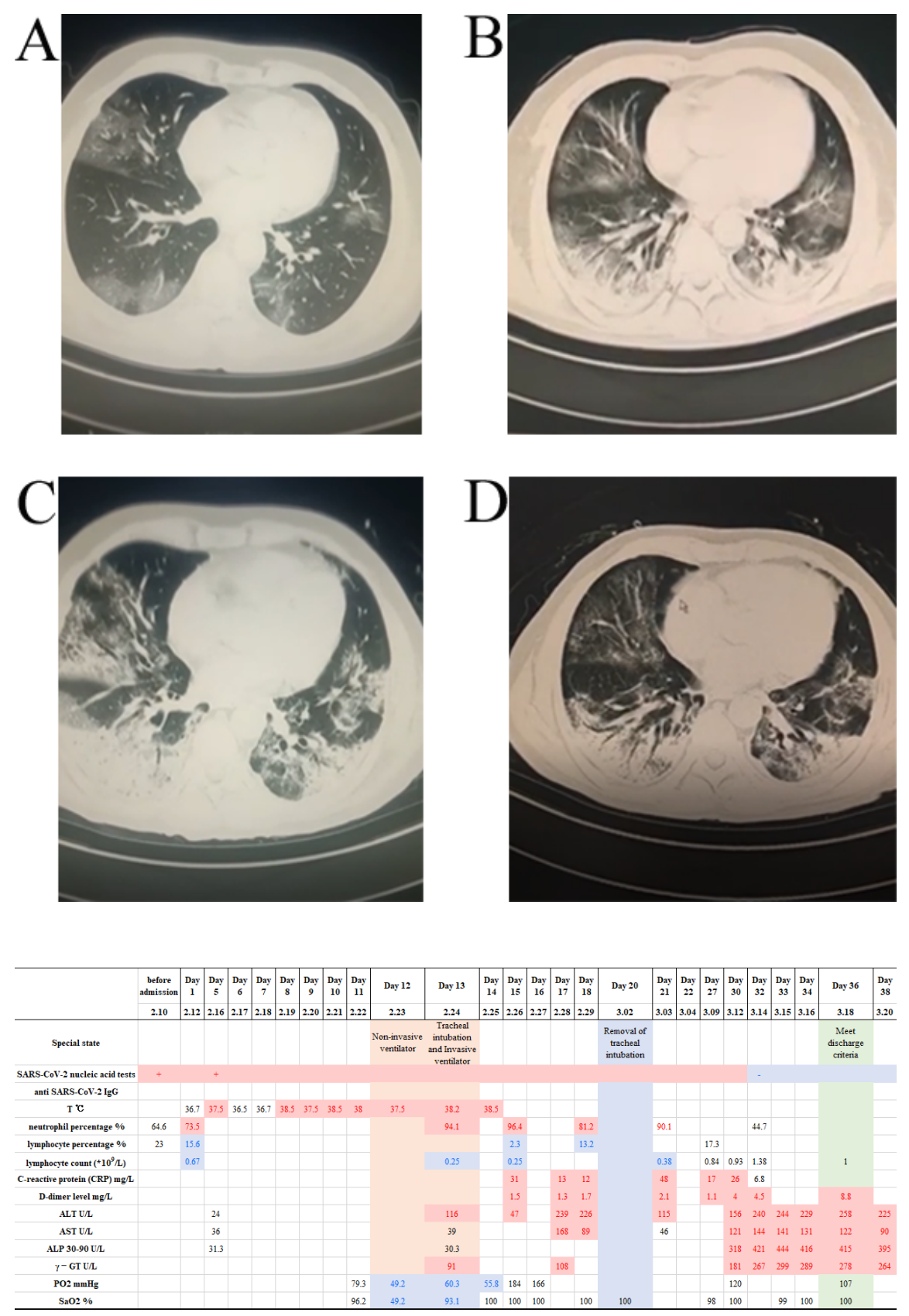